\title{
STM-SEM COMBINATION STUDY ON THE ELECTROCHEMICAL GROWTH MECHANISM AND STRUCTURE OF GOLD OVERLAYERS
} A quantitative approach to electrochemical metal surface roughening

\author{
L. VAZQUEZ, A. BARTOLOME, A.M. BARO \\ Departamento de Fisica de la Materia Condensada, Universidad Autonoma de Madrid, \\ 28049 Madrid, Spain
}

C. ALONSO, R.C. SALVAREZZA *

Departamento de Quimica, Universidad Autonoma de Madrid, 28049 Madrid, Spain

and

\author{
A.J. ARVIA \\ INIFTA, Unitersidad Nacional de La Plata, Sucursal 4, Casilla de Correo 16, \\ 1900 La Plata, Argentina
}

Received 30 September 1988; accepted for publication 20 January 1989

The growth mode and structure of gold overlayers resulting from the electroreduction of thick oxide films are studied using potentiodynamic and potentiostatic techniques combined with ex-situ STM and SEM. Electroreduction of the thick gold oxide at low overpotentials, that is slowly grown gold overlayers, results in a close-packed array of grains exhibiting a low roughness factor. Grains in the order of $100 \mathrm{~nm}$ of radii are formed by aggregation of small monomers. At higher overpotentials, fast grown gold overlayers, we suggest that the monomer growth results in a columnar structure terminated on rounded domes with radii ranging between 10 and $20 \mathrm{~nm}$. This overlayer exhibits a roughness factor which increases according to electrodeposit height. The columnar structure is unstable decreasing its surface free energy by coalescence of small columns to form large units leading to a drastic decrease in the surface area with ageing time. A mechanism for the growth mode is proposed where the crystallite size depends on the diffusion length of the electrodepositing particles which is controlled by the applied overpotential.

\section{Introduction}

The active surface area and electrochemical properties of metal overlayers like platinum, gold, silver etc, produced by electroreducing thick oxides layers which were accumulated by applying relatively fast periodic potentials in acid electrolytes, can be easily modified by using different electroreduction conditions, i.e. a potential sweep at a certain scan rate or a constant potential step

* Permanent adress: INIFTA, La Plata, Argentina. 
[1-4]. In the case of gold overlayers it was established that the surface roughness increases markedly according to the rate of the oxide to metal phase change. Recently, the growth modes of gold overlayers were simulated by means of the Monte Carlo method [5]. Smooth and rough metal overlayers can be obtained depending on whether the oxide layer electroreduction is controlled by the metal ion attachment itself or by the transport of metal ions in the metal oxide layer. Accordingly, the grain size of the metal overlayer should depend on the diffusion path of the electrodepositing particles as proposed for metallic growth from the vapor phase [6].

Although an important amount of work on this field has been done by using macroscopic techniques like voltammetry, it is necessary to measure the microscopic structure in order to gain insight on the physicochemical processes which are behind the observed results.

It should be noticed that electrochemical measurements give indirect information about the properties of the resulting structures such as preferred orientation and roughness. The latter reflects mainly average geometric parameters of crystallites i.e. mean radius and height which must be measured directly by microscopy. In many case the particle size lies in the nanometer region and novel microscopies such as STM are required to characterize the metal structure [7-10]. However, rough surfaces are frequently heterogeneous, so that STM results which are limited to $-1 \mu \mathrm{m} \times 1 \mu \mathrm{m}$ scan, fail to give representative data of the electrode. For this type of surfaces, the combination between STM and SEM seems to be a promising tool because it allows one to select a characteristic region of the specimen by SEM and then study it at the nanometer level with the STM.

Since the invention of the STM, this combination has been tried by several groups with different success [11-13]. Recently, we have designed a combined STM-SEM microscope [14] which has been suitable to study rough surfaces [15].

In this paper, the combined STM-SEM microscope is used to study the structure of gold overlayers resulting from the electroreduction of thick oxides and their time evolution in aqueous acid electrolytes. A close correlation between the two microscopies is found in the submicrometer scale whereas STM provides a detailed picture of the electrodeposits in the nanometer range which is not accessible to SEM. This information is relevant to interpret electrochemical data about structure, growth mode and stability of the electrodeposits. A model for the growth of these overlayers is also presented in analogy with the growth of metal from the vapor [6].

\section{Experimental}

The electrochemical system included a polycrystalline gold wire working electrode, a large gold counter electrode, a mercury/mercureous sulphate 
reference electrode and a solution of $0.5 \mathrm{M} \mathrm{H}_{2} \mathrm{SO}_{4}$ placed in a three compartment glass cell. The potential values are referred to the standard hydrogen electrode (SHE). The thick oxide layer electroformation was made by using the well known repetitive square wave perturbing potential (RSWPP) method described in the literature [16] or by anodizing the gold electrode at a constant potential, $E_{\mathrm{a}}\left(E_{\mathrm{a}}=2.44 \mathrm{~V}\right)$ during a certain time $t_{\mathrm{a}}$. The RSWPP was applied at a frequency of $4 \mathrm{kHz}$, the lower and the upper potentials being fixed at 0.6 and $3.1 \mathrm{~V}$ respectively. The gold overlayer was obtained from the potentiostatic elcctroreduction of the thick gold oxide layer [3]. Immediately there after, the evaluation of the electrode roughness was made by using the voltammetric method [9]. Then, the ex-situ SEM-STM pictures of the different specimens were obtained.

To obtain the STM images of highly corrugated specimens, special care was taken to select the tip. For these topographies, the macroscopic shape of the tip is important for a good quality image as already verified [15]. In this case the combined use of SEM and STM has been of great help in order to test radius and tip shape.

In all cases, the measurement procedure was the same. Due to the surface heterogeneity, the SEM was used to address the tip to a representative region of the specimen. Then, the STM data were taken. Afterwards, the sampled region was identified and the SEM pictures were obtained.

The STM data are presented in two different ways: (i) a three-dimensional representation done by lateral shadowing and (ii) a contour level representation. Due to the difference in height between grains the STM contour level representation produces a low contrast at small grains washing out those grains smaller than the grey level step.

The SEM pictures in combination with STM are obtained at an angle of $45^{\circ}$ between the specimen and the electron beam.

\section{Results}

\subsection{Electrochemical data}

The kinetics of the electrochemically induced phase change from the thick gold oxide to the metal gold overlayer depends on the overpotential, $\eta$, defined as the difference between the reversible potential, $E_{\mathrm{r}}$ of the $\mathrm{Au} / \mathrm{Au}_{2} \mathrm{O}_{3}$ redox couple $\left(E_{\mathrm{r}}=1.36 \mathrm{~V}\right.$ SHE) [18], and the applied potential. When the electroreduction of the thick oxide layer proceeds at a constant value of $\eta$, the current density-time curves exhibit a maximum, $J_{M}$ (at the time $t_{M}$ ) indicating that the rate controlling step involves nuclei formation followed by nuclei growth [3,19-22] (fig. 1a). For a constant amount of thick oxide i.e. a constant electroreduction charge density, $q$, the increase in $\eta$ for $0.38 \mathrm{~V}<\eta<0.60 \mathrm{~V}$ 


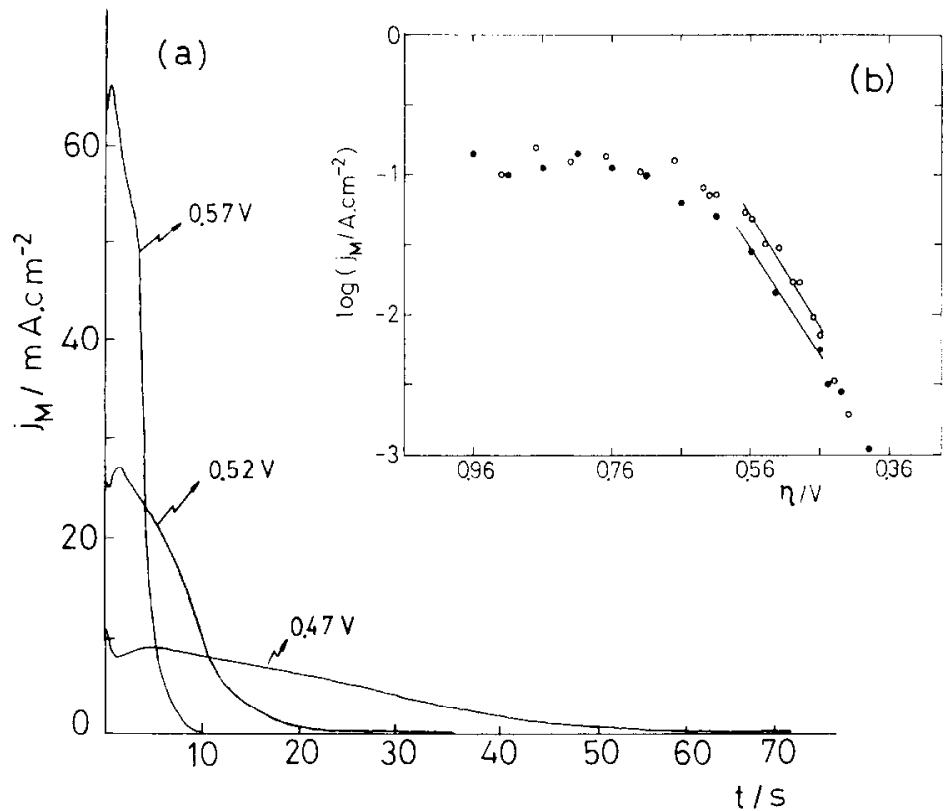

Fig. 1. (a) Current transients at constant $\eta$ related to the electroreduction of the gold oxide layer formed at $E_{\mathrm{a}}=2.44 \mathrm{~V}$ and $t_{\mathrm{a}}=120 \mathrm{~s}, q=0.3 \mathrm{C} \mathrm{cm}^{-2}$. (b) $\log J_{\mathrm{M}}$ versus $\eta$ plot resulting from the potentiostatic electroreduction of oxide gold layers: $(\bullet)$ oxide layer accumulated by RSWPP method (ref. [3]) $t_{\mathrm{a}}=60 \mathrm{~s}$. (O) oxide layer formed at $E_{\mathrm{a}}=2.44 \mathrm{~V}$ and $t_{\mathrm{a}}=120 \mathrm{~s} . T=298 \mathrm{~K}$.

results in a progressive increase in the rate of the phase change until the rate of this process approaches a constant value for $\eta>0.6 \mathrm{~V}$. Thus, in the 0.38 $\mathrm{V}<\eta<0.6 \mathrm{~V}$ range the $\log J_{\mathrm{M}}$ versus $\eta$ plot exhibits a Tafel behaviour with a slope close to $0.12 \mathrm{~V} /$ decade whereas for $\eta>0.6 \mathrm{~V}, J_{\mathrm{M}}$ becomes practically independent of $\eta$ as one should expect when the rate of the overlayer growth becomes limited by the rate of mass transport in the oxide phase (fig. 1b).

The gold overlayers produced at the lower $\eta$ values (condensed gold overlayers), exhibit a small surface roughness factor, $R$ (fig. 2) which can be defined as the overall active surface area per square centimeter of substrate. The $R$ value was measured as the ratio of the voltammetric electroreduction charge of the $\mathrm{O}$-monolayer formed on the gold overlayer to that formed on the polycrystalline gold electrode before the thick oxide layer accumulation treatment [9].

As $\eta$ increases for 0.38 to $0.60 \mathrm{~V}$ and the rate of the gold overlayer electroformation becomes larger, $R$ increases markedly to reach a value which depends on the initial amount of oxide. In this range of $\eta$, the $\log R$ versus $\eta$ plot gives also a straight line with a slope close to $0.24 \mathrm{~V} /$ decade (fig. 2). 


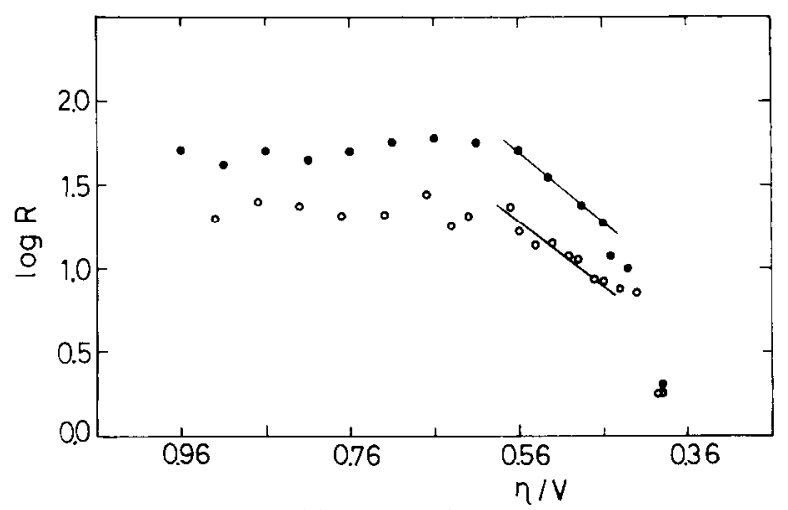

Fig. 2. $\log R$ versus $\eta$ plots. (๑) Resulting from the potentiostatic electroreduction of a gold oxide layer formed through the RSWPP method for $t_{\mathrm{a}}=60 \mathrm{~s}$ (ref. [3]), $(0)$ oxide layer formed at $E_{\mathrm{a}}=2.44 \mathrm{~V}$ and $t_{\mathrm{a}}=120 \mathrm{~s}, T=298 \mathrm{~K}$.

Finally for $\eta>0.6 \mathrm{~V}$ that is for fastest grown overlayers (activated gold overlayers) $R$ remains practically constant.

It should be noticed that the RSWPP procedure to form the oxide gold layer yields large $R$ values than the potentiostatic method (fig. 2).

At a constant $\eta$, for $\eta>0.60 \mathrm{~V}, R$ increases linearly with increasing $q$, that is the amount of oxide accumulated on the electrode, the slope of the straight line being independent of $\eta$ in the $0.60-0.92 \mathrm{~V} \eta$ range (fig. 3). Conversely for small values of $\eta, R$ becomes close to 2 and is independent of $q$.

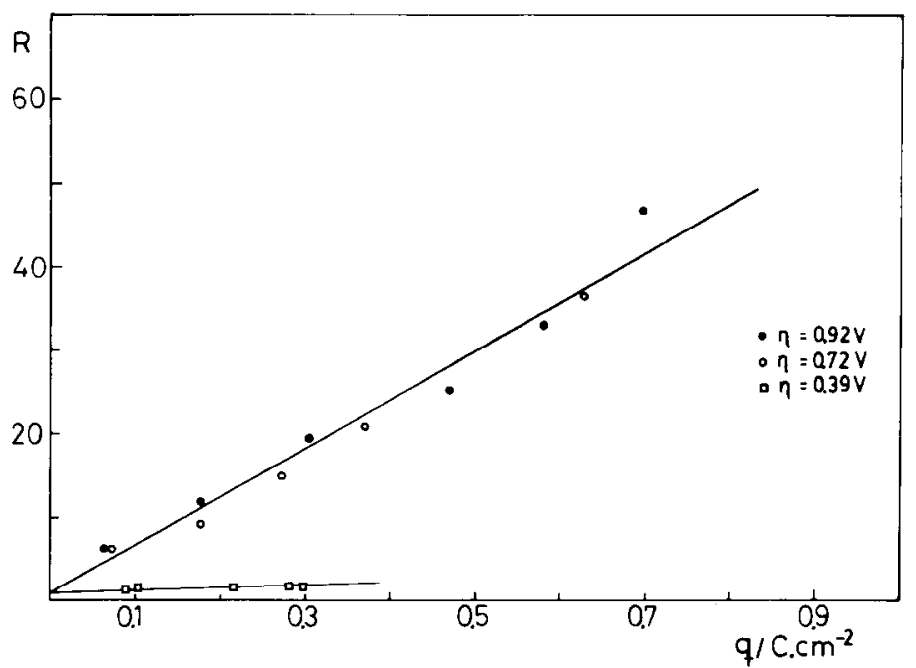

Fig. 3. $R$ versus $q$ plots for different $\eta$ values. 


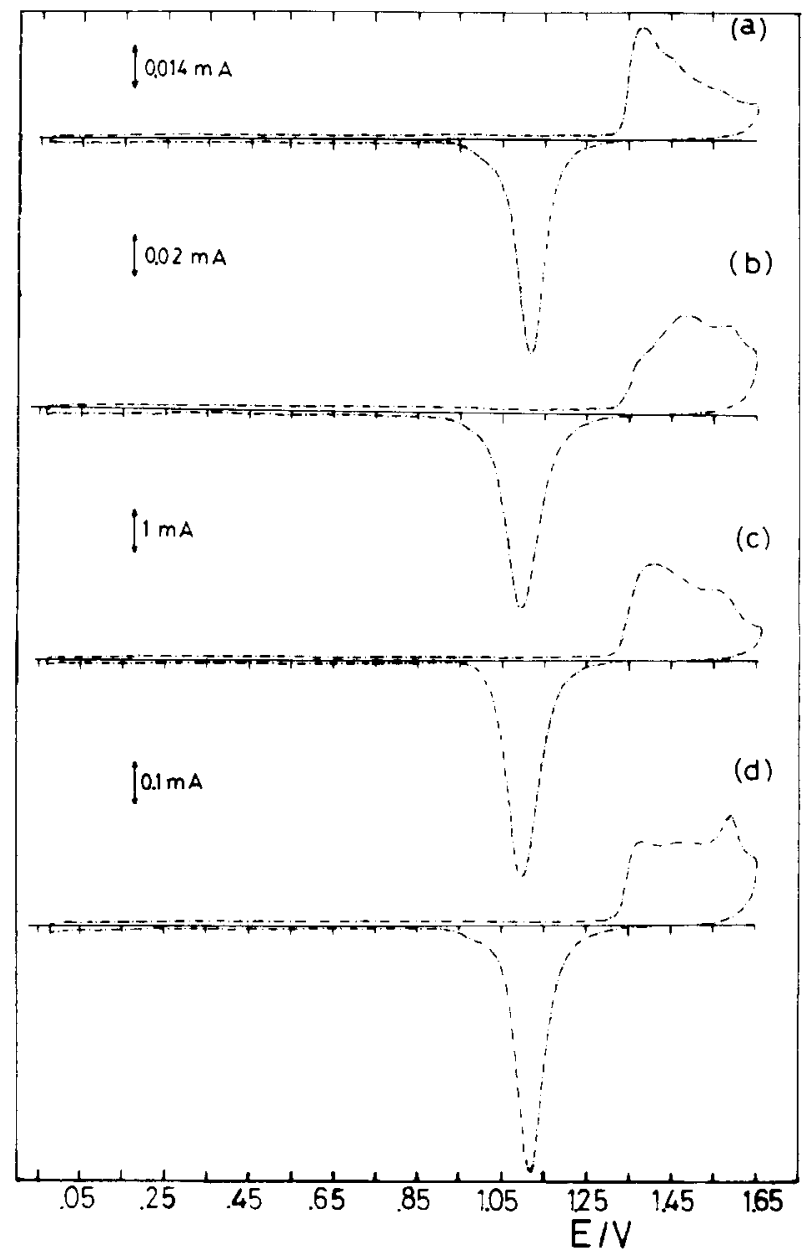

Fig. 4. Voltammograms at $0.1 \mathrm{~V} / \mathrm{s}$ between $E_{\mathrm{s}, \mathrm{a}}=1.65 \mathrm{~V}$ and $E_{\mathrm{s}, \mathrm{e}}=0.05 \mathrm{~V}$ in $0.5 \mathrm{M} \mathrm{H}_{2} \mathrm{SO}_{4}$ at 298 $\mathrm{K}$ for (a) polycrystalline untreated gold electrode, (b) gold overlayer resulting at $\eta=0.376 \mathrm{~V}$, (c) gold overlayer produced at $\eta=0.92 \mathrm{~V}$, voltammetry made immediately after the gold oxide electroreduction process was finished, (d) gold overlayer made at $\eta=0.92 \mathrm{~V}$, voltammogram run after the gold overlayer was aged during $10^{4} \mathrm{~s}$ under open circuit in $0.5 \mathrm{M} \mathrm{H}_{2} \mathrm{SO}_{4}$ at $T=325 \mathrm{~K}$.

The activated overlayers exhibit a decay in $R$ with time (relaxed gold overlayers) either in air or in $0.5 \mathrm{M} \mathrm{H}_{2} \mathrm{SO}_{4}$ at open circuit whereas no change in $R$ can be detected for the polycrystalline untreated and condensed gold overlayers whose voltammograms remain unaltered after ageing. It can be noticed that the large differences in the voltammogram arise from the charge involved which is specifically due to the roughness of the electrodes and also 


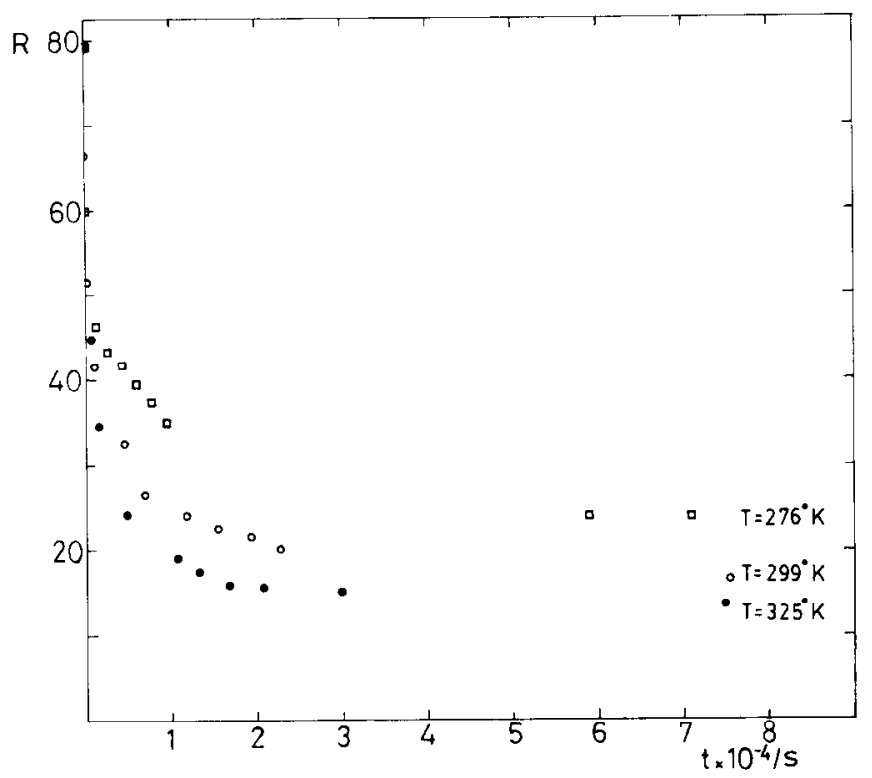

Fig. 5. Experimental $R$ versus $t$ plots at different temperatures.

from certain preferred crystallographic orientation which has been developed for the condensed gold overlayers (figs. $4 \mathrm{a}$ and $4 \mathrm{~b}$ ).

The decay in $R$ of the activated gold overlayer is faster as the temperature, $T$, of the system is increased from 276 to $325 \mathrm{~K}$ (fig. 5). In all cases, a progressive change in the shape of the voltammograms accompanies the decay in $R$ (figs. 4 c and $4 \mathrm{~d}$ ). Thus, the relative contribution of the voltammetric peaks related to the O-monolayer electroformation is drastically changed by increasing the contribution of the current peak located more positively. After, ageing the activated gold overlayer for $10^{4} \mathrm{~s}$, the voltammogram resembles that reported for $\mathrm{Au}(111)$ in acid solutions [23,24] (fig. 4d). Finally, the voltammogram recorded after an ageing of $6 \times 10^{4} \mathrm{~s}$ is similar to that recorded for the slowly formed gold overlayer (fig. $4 \mathrm{~b}$ ).

\subsection{Microscopic observations}

3.2.1. Data from the activated, condensed and relaxed gold overlayers obtained by the use of STM in combination with SEM

(1) The activated gold overlayers correspond to those obtained by electroreduction at high $\eta$. The electrodeposits are characterized by a large value of $R$ which depends on $q$. SEM images of these specimens reveal the presence of small grains which are in the limit of SEM resolution for this kind of specimen (fig. 6a). The image shows also channels of about $300 \mathrm{~nm}$ width. Along the 

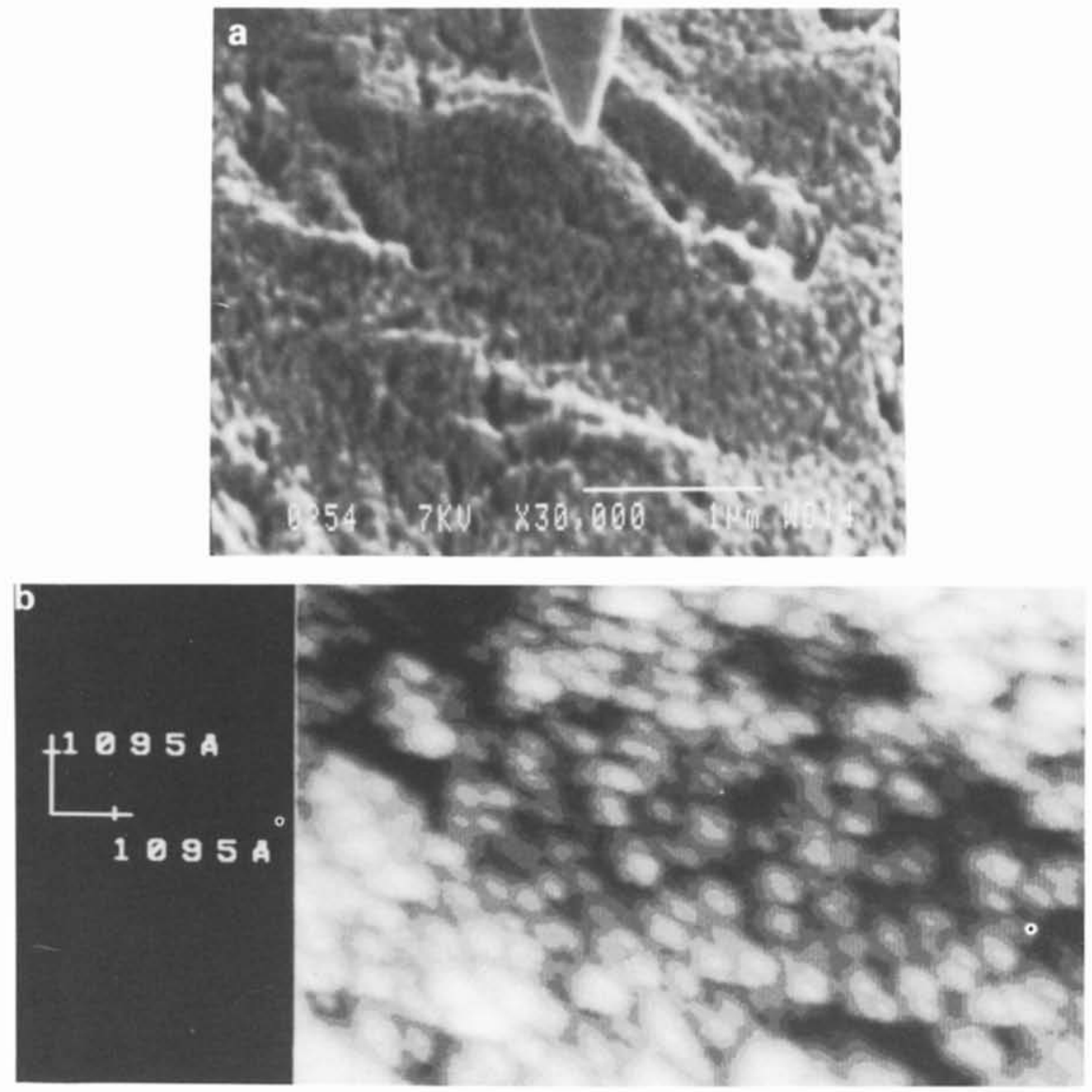

c

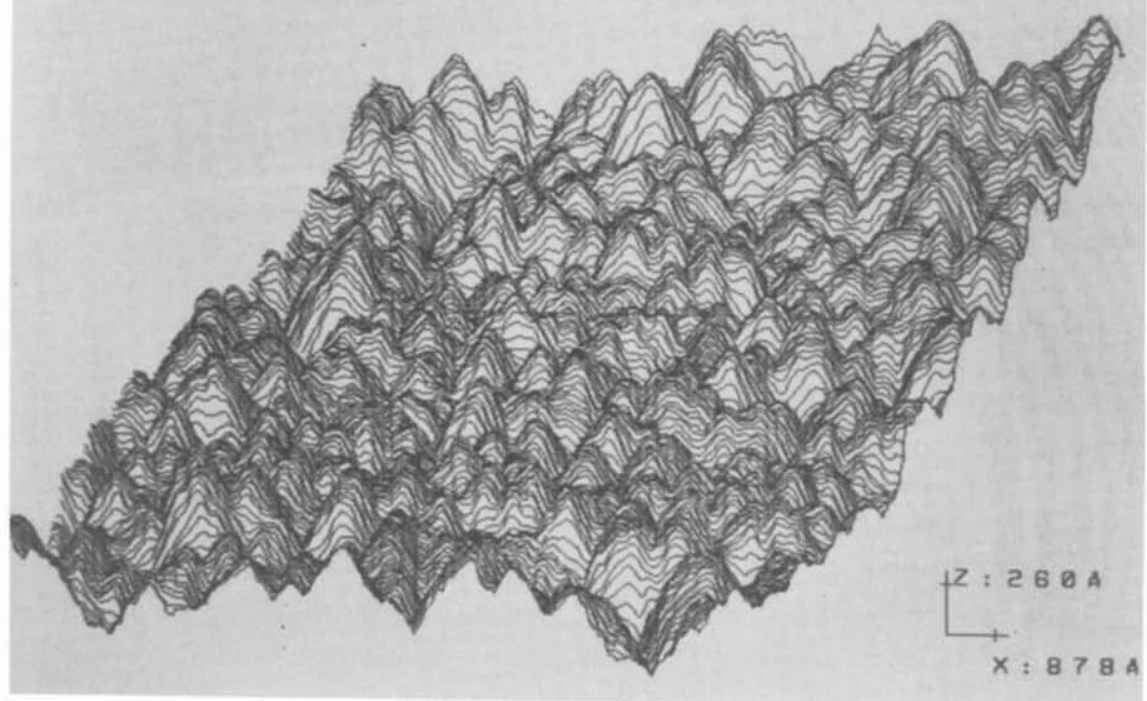


channels it is possible to see at the structure of those grains below the surface. These images already furnish some indication of columnar growth.

In the STM images (figs. $6 \mathrm{~b}$ and $6 \mathrm{c}$ ) the grains are clearly resolved. From fig. $6 \mathrm{~b}$ one can obtain the size distribution plot which yields a mean value close to $30 \mathrm{~nm}$ and a grain density, $N$, between $10^{10}$ and $10^{11} \mathrm{~cm}^{-2}$. The STM image reveals also less dense structures and big holes which are close to 300 $\mathrm{nm}$ across. The three-dimensional representation of the STM data allows us to observe the fine structure of these grains (fig. $6 \mathrm{c}$ ). One can find that the grains are flat with a characteristic dome structure on top as already reported [9]. It should be noticed that some big grains are formed by two or three small units.

(2) The condensed gold overlayers have been obtained by electroreduction of the gold oxide layer at a low $\eta$. The corresponding $R$ value is very low $(R=2)$ and independent of $q$.

The SEM images show a grain structure with grain size ranging between 100 and $200 \mathrm{~nm}$ (fig. 7a). The STM image has been taken at the same place (figs. $7 \mathrm{~b}$ and $7 \mathrm{c}$ ). A close correspondence of features on the two images is found. In order to guide the eye a square on the SEM image has been drawn. There is a characteristic bright spot on this region which is marked with an arrow on the STM image. It can be noticed that the length of the main channel (aligned in the arrow direction) seems to be smaller than in the SEM image. This fact can be explained considering that the channel depth seen by the tip is not constant so that the colour is not the same along it whereas it appears as a completely dark structure in the SEM.

The STM image shows clearly the differences in grain size between the two specimens: the activated and the condensed one. But the more important point about the STM image is that the grains observed by SEM are formed by groups of two, three and four monomers ( $50 \mathrm{~nm}$ size). Even more, some of these grains aggregate together to form larger structures up to $1 \mu \mathrm{m}$ size. This coalescence process is the most important feature of the specimen. From fig. $7 \mathrm{~b}$, the monomer density is close to $10^{10} \mathrm{~cm}^{-2}$. The three-dimensional representation (fig. 7c) shows a smoother surface with more linked grains than that observed for the activated specimen. The aggregation process is not visible in the SEM image. This is probably due to the lack of SEM vertical resolution and/or to the so-called shadow contrast which would only produce brightness on the most prominent structures. A more detailed discussion of STM versus SEM imaging will be the object of a forthcoming publication [25]. Sometimes, preferred orientations of grains on the surface of the sample are detected in the STM image.

Fig. 6. (a) SEM micrograph of a gold overlayer made at $\eta=0.92 \mathrm{~V}, R=70$. On the upper part the tip used for STM is imaged. (b) Countour level plot. The grey-tone scale range is $3.36 \mathrm{~nm} /$ level. Image of $1.1 \mu \mathrm{m} \times 0.82 \mu \mathrm{m}$ area. (c) $3 \mathrm{D}$ processed image of the same data as in (b). Tunnel current: $1 \mathrm{nA}$, tunnel voltage: $0.86 \mathrm{~V}($ tip + ). 


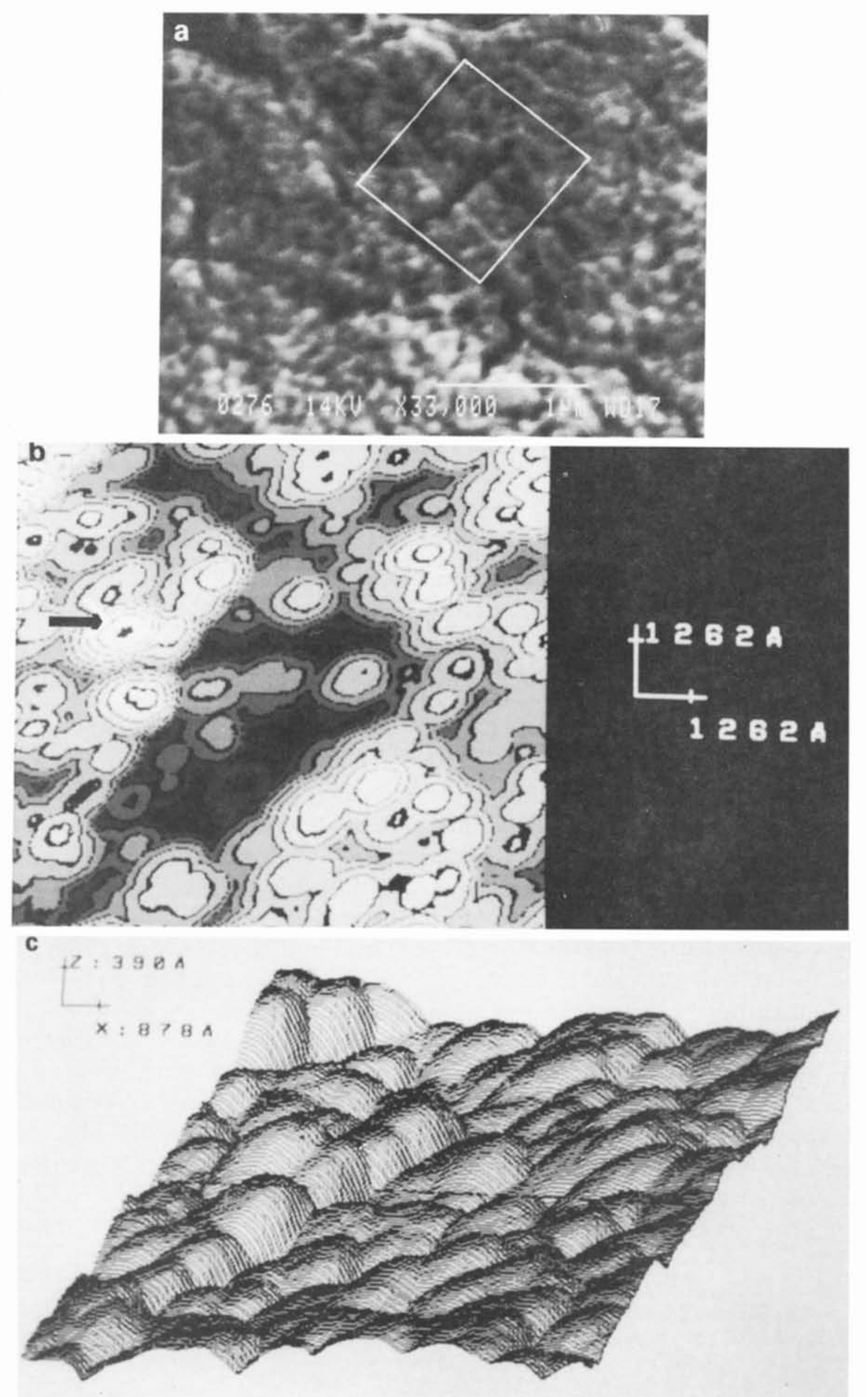


(3) The relaxed gold overlayers have been initially produced as the activated specimens and afterwards they have been left at open circuit in $0.5 \mathrm{M} \mathrm{H}_{2} \mathrm{SO}_{4}$ at $325 \mathrm{~K}$. For one specimen ageing during $6 \times 10^{4} \mathrm{~s}$, a decrease in $R$ from 70 to 10 has been observed.

The corresponding SEM images (fig. 8a) show the brain-like aspect which has been reported previously as characteristic of the gold overlayers resulting from the activation process [9]. In fact the more characteristic features are the voids and not the grains. One should point out that this brain-like aspect appears even for relatively small $R$ valucs. It is clear that this topographic structure is not responsible for the large values of $R$.

The relaxed specimen provides also the best opportunity to make a correlation between SEM and STM data as the present instrumentation used throughout this work allows one to image each specimen simultaneously at the two levels. Figs. 8a and $8 \mathrm{~b}$ show an example of this correlation. Here the correlation can be made by using the voids present on the surface because the grains are poorly resolved in the SEM micrograph. Every big void appearing in both images can be identified. From the big voids, the small ones which appear with a minor contrast in the STM contour level image are also identified. As was already done for the condensed sample a square in the SEM image was drawn in order to show the borders of the region scanned by the STM. The better resolution of STM can be detected by the sharpness of the channel walls.

Looking now at the STM image, it shows a distribution of grains with size very similar to that found for the condensed specimen (fig. 8b). An aggregation process of monomers forming structures of $200 \mathrm{~nm}$ in size can also be observed. The shape of the individual grains is different, as for the relaxed specimens they appear as terminated on a hemispherical dome (fig. 8c) whereas for the condensed specimen they appear flat (fig. 7c).

\subsubsection{Independent SEM observations}

The structure of the activated gold overlayer which was obtained from a cross section of the electrode shows a clear contrast between the overlayer and the substrate. For $q=1.2 \mathrm{C} \mathrm{cm}^{-2}$, the electrodeposit height, $h$, is close to 800 $\mathrm{nm}$. In addition the structure of the overlayer seems to be consistent with the presence of columns as already mentioned.

Furthermore the SEM image of the gold oxide layer itself contains grains of $10 \mu \mathrm{m}$ size which indicates that none of the structure reported before like

Fig. 7. (a) SEM micrograph of a gold overlayer obtained at $\eta=0.376 \mathrm{~V}, R=2$. (b) Contour level plots. The grey-tone scale range is $6.36 \mathrm{~nm} /$ level. Image of $0.94 \mu \mathrm{m} \times 0.93 \mu \mathrm{m}$ area. (c) $3 \mathrm{D}$ processed image of the same data as in (b). Fig. $7 \mathrm{~b}$ is taken on the region marked with a white square in fig. 7a. The main channel in the same image aligned with the arrow shows the brightest spot on both images. Tunnel current: $3 \mathrm{nA}$, tunnel voltage: $0.12 \mathrm{~V}$ (tip +). 
channels can be related to the oxide layer. Attempts to obtain tunneling between the tip and the oxide or between the tip and a partially reduced sample $(1 / 3)$ gave negative results indicating that the gold overlayer starts to grow from the metal/oxide interface outwards. It should be noticed that the
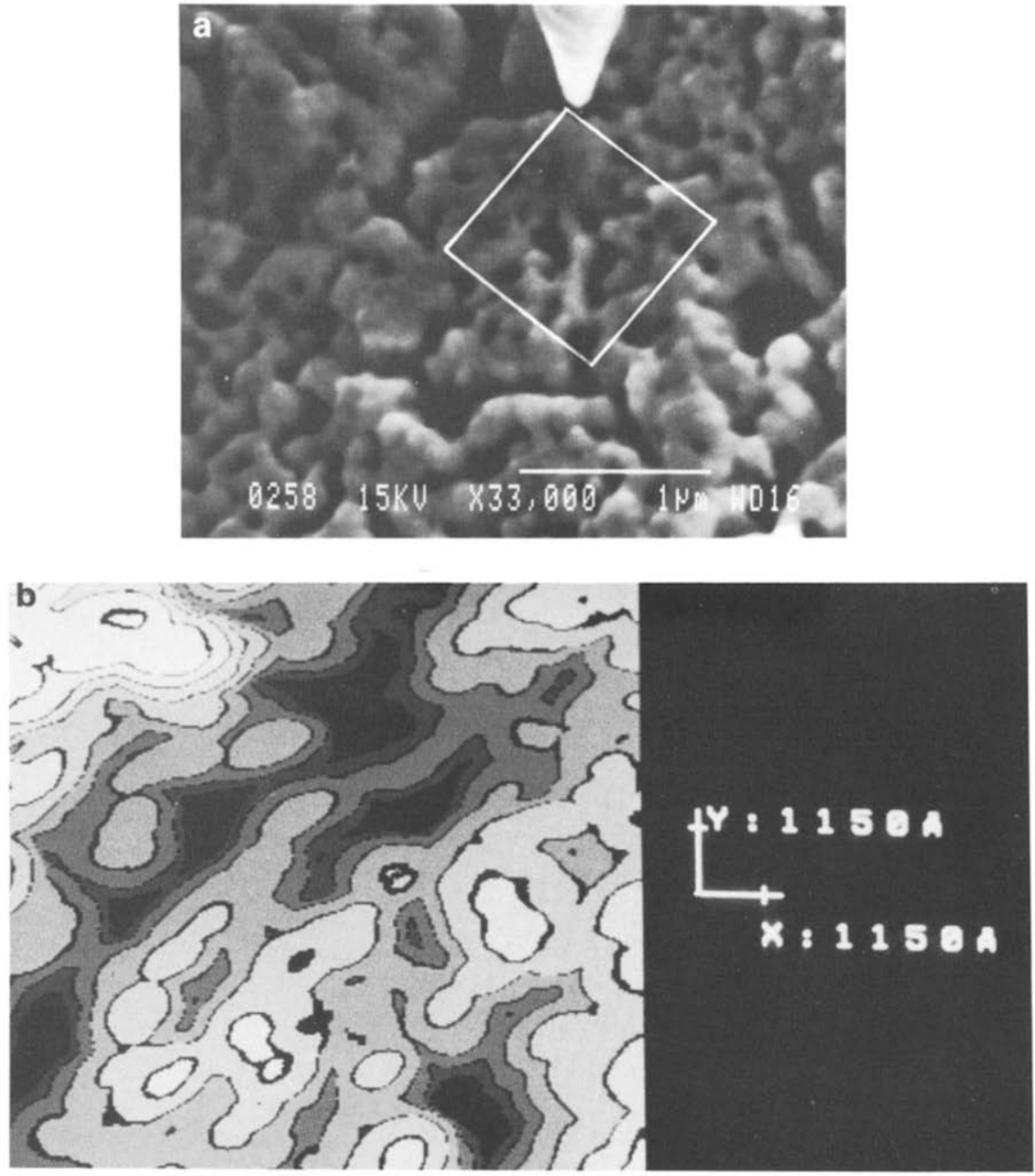

Fig. 8. (a) SEM micrograph of a gold overlayer formed at $\eta=0.92 \mathrm{~V}$ after ageing during $6 \times 10^{4} \mathrm{~s}$ in $0.5 \mathrm{M} \mathrm{H}_{2} \mathrm{SO}_{4}$ at operl circuit $(R=10)$. $T-325 \mathrm{~K}$. (b) Contour level plot corresponding to the square region in (a). The grey-tone scale range is $10.1 \mathrm{~nm} /$ level. Image of $0.84 \mu \mathrm{m} \times 0.90 \mu \mathrm{m}$ area. (c) 3D processed image of the same data as in (b). The better resolution of STM data can be noticed from the sharp profile of void walls. Tunnel current: $3 \mathrm{nA}$, tunnel voltage: $0.54 \mathrm{~V}($ tip + ). 


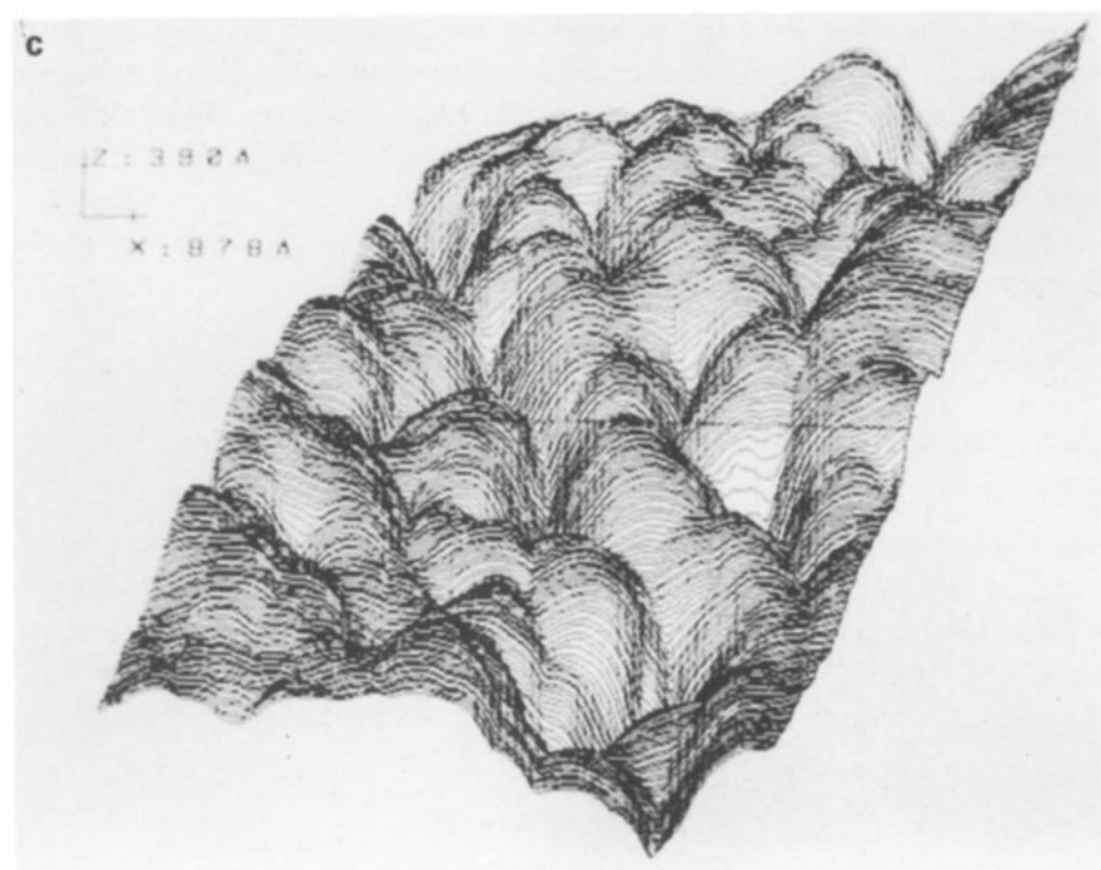

Fig. 8. Continued.

oxide acts as a too poor conductor to allow electron tunneling under the present conditions.

\section{Discussion}

A model for the structure and growth mode of metal overlayers obtained from the electroreduction of thick oxides must be consistent with direct STM-SEM observations and electrochemical data. The relevant experimental information may be summarized as follows:

Electrochemical kinetics data indicate that the electroreduction process is controlled by nuclei formation followed by growth.

Nuclei are formed at the metal/oxide interface because no tunneling current between the tip and a partially reduced specimen is detected.

STM observation shows that monomer size and density do not change appreciably when $\eta$ is increased. It implies that an instantaneous nucleation operates at the early stage of the electroreduction process.

The combined STM-SEM data reveal that $\eta$ influences markedly the growth process. At low $\eta$, monomers aggregate forming large units and the 
structure can be described by an array of hemispherical crystallites. On the other hand at the high $\eta$ values monomers grow mainly as isolated islands and the microscopic observation suggests a columnar structure. Other structures, however, may be compatible with the observed $R$ values i.e. the sticking sphere model.

In the next, and at the light of the experimental information, we develop with greater detail two particular points.

\subsection{The relationship between $R$ and the proposed structure}

According to definition, $R$ is given by

$R=s N+s^{\prime} N^{\prime}$,

where $s$ is the average area of a 3D grain, $N$ is the grain density $\left(\mathrm{cm}^{-2}\right), s^{\prime}$ is the area of noncovered units of the substrate (voids between grains) and $N^{\prime}$ is the void density $\left(\mathrm{cm}^{-2}\right)$. For a $3 \mathrm{D}$ electrodeposit formed by a rectangular array of columns of average height, $h^{\prime}$, and average radii $r$ terminated with a hemispherical dome of the same radius, of $\mathrm{C}_{4 v}$ symmetry, one obtains

$s=2 \pi r h^{\prime}+2 \pi r^{2}$,

$N=1 / 4 r^{2}$,

$s^{\prime}=(4-\pi) r^{2}$,

$N=N^{\prime}$.

Introducing eqs. (2)-(5) into eq. (1), we obtain:

$R=(\pi / 2 r)\left(h^{\prime}+r\right)-\pi / 4+1$,

the total average height of electrodeposit, $h$, is given by:

$h=h^{\prime}+\frac{2}{3} r$,

where $\frac{2}{3} r$ is the average height of the hemispherical top dome. Thus,

$R=(\pi / 2)(h / r)-(\pi / 12)+1$.

For $h \gg r$

$R=(\pi / 2)(h / r)$.

Expression (6) includes the particular case where the development of a rectangular array of hemispherical grains takes place. In this case, $h^{\prime}=0$ and, therefore

$R=\pi / 2-\pi / 4+1=1.8$.

We can also obtain an other expression for $R$ by assuming that grains are spheres of radius $r$ packed in the same way as in the fcc crystal structure. Then for $h \gg r$ one obtains

$R=(\pi / \sqrt{2})(h / r)$. 
Eq. (11) gives for the same value of $r$ a value of $R$ larger than that coming out from expression (9).

Thus, eqs. (9), (10) and (11) can be used to investigate the structure of the metal overlayer, provided that the values of $h$ or $r$ are known.

The $h$ values corresponding to the gold overlayers can be estimated from electrochemical data. The electroreduction of the gold oxide layer to form the gold overlayer can be represented by the following equation:

$\mathrm{Au}_{2} \mathrm{O}_{3}+6 \mathrm{H}^{+}+6 \mathrm{e}^{-} \rightarrow 2 \mathrm{Au}+3 \mathrm{H}_{2} \mathrm{O}$.

Both the thickness of the oxide, $h_{\mathrm{ox}}$, and, the thickness of the metal overlayer, $h$, can be estimated from the equation:

$h=M q / z F \rho$,

where $M$ and $\rho$ are the molecular weight and the density of the electrodeposit. Thus for $\mathrm{Au}_{2} \mathrm{O}_{3}$ electrodeposit by taking $M=442 \mathrm{~g} / \mathrm{mol}, z=6, \rho=11 \mathrm{~g} / \mathrm{cc}$ [26] and $q=1.2 \mathrm{C} \mathrm{cm}^{-2}, h_{\text {ox }}$ becomes $8.2 \times 10^{-5} \mathrm{~cm}$. Similarly, for Au electrodeposit from eq. (13) and taking $M=197 \mathrm{~g} / \mathrm{mol}, z=3, \rho=19 \mathrm{~g} / \mathrm{cc}, h$ becomes close to $4.3 \times 10^{-5} \mathrm{~cm}$. These results indicate that if the phase change proceeds to form a condensed overlayer, $h \simeq h_{\text {ox }} / 2$. Conversely when the phase change proceeds at a constant apparent volume (the oxide volume) $h \simeq h_{\text {ox }}$, and, then a low density overlayer is formed. This last condition, seems to be approached for the activated gold overlayer, which exhibits a large number of voids and channels (fig. 6). Thus, SEM data of the activated overlayer cross section reveal that $h$ is close to the $h_{\text {ox }}$ value calculated with eq. (13). Simple volume consideration indicates that for the close-packed array of grains, $h \simeq 0.66 h_{\mathrm{ox}}$. It implies that eqs. (9) and (11) must be modified in order that the $h=h_{\text {ox }}$ condition is fulfilled. Thus, a factor $2 / 3$ should be introduced into eq. (9) to obtain

$R=(\pi / 3)(h / r)$

for the columnar structure, and

$R=(2 \pi / 3 \sqrt{2})(h / r)$

for the spheres model. The $2 / 3$ factor accounts for the voids and channels present in the structure.

Introducing eq. (13) into eqs. (14) and (15) one obtains

$R=(\pi / 3)(k q / r)$

and

$R=(2 \pi / 3 \sqrt{2})(k q / r)$

for the columnar and spheres models, respectively. In eqs. (16) and (17) $k=M / z F \rho$. It is clear from fig. 3 that the $R$ versus $q$ plot gives a straight line at high $\eta$, whereas $R \simeq 2$ and independent of $q$ at low $\eta$ values. Thus, the 
structure of the slowly grown metal overlayer can be satisfactorily described by a close-packed array of hemispherical grains, as for this model $R=1.8$ (eq. (10)) in excellent agreement with the experimental data. On the other hand, the columnar and sphere models can be applied to the fast grown overlayers. The slope of the $R$ versus $q$ linear plot yields $r=12.3 \mathrm{~nm}$ for eq. (16) and $r=17.4 \mathrm{~nm}$ for eq. (17). Both values are in reasonable agreement with the average grain size resulting from the STM images considering that the real size should be slightly smaller due to the broadening produced by tip shape. The SEM and STM data favour the columnar structure as the most adequate one to describe the metal overlayer. Besides, for the sphere model it would expect an instantaneous and homogeneous nucleation in the metal oxide phase adjacent to the metal phase. This fact appears to be rather improbable because the thick layer acts as a poor conductor for electron tunneling. Otherwise, if nucleation would occur in the whole oxide volume the current transient parameters, $J_{\mathrm{M}}$ and $t_{\mathrm{M}}$ would increase as the oxide layer becomes thicker due to the larger number of sites available for nucleation. This is opposite to the experimental facts, where, both $J_{\mathrm{M}}$ and $t_{\mathrm{M}}$ remain practically constant as $h_{\mathrm{ox}}$ increases.

\subsection{The development of a growth model for the columnar structure}

The mechanism involved in the electroformation of this structure should be interpreted by using the model for columns grown during metal deposition from the vapor phase [6]. In this model, the most probable column diameter, $2 r$, is related to the mean surface diffusion distance, $x$, of a single depositing particle before its attachment to the growing surface. Thus

$2 r=2 \pi x$

and by introducing eq. (18) into eq. (14) one obtains

$R=(h / 3 x)$.

On the other hand, $x$ is related to the residence time of the particle on the growing surface before its attachment [6] by the relationship

$x^{2}=2 D t$,

where $t$ is related to the sticking frequency, $A(\eta)$ by

$A=1 / t$.

Thus, from eqs. (20) and (21) eq. (19) transforms into

$R=h \sqrt{A} / 3 \sqrt{2 D}$.

$A$ can be obtained from the columnar growth rate, $\partial h / \partial t$, through the expression in ref. [6]:

$\rho \partial h / \partial t=A c$, 


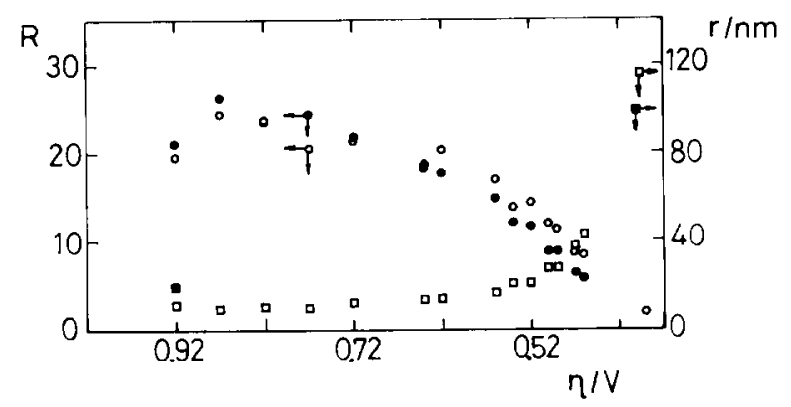

Fig. 9. $R$ versus $\eta$ plot. (O) Experimental data, (๑) calculated data from eq. (26) and $D=7 \times 10^{-12}$

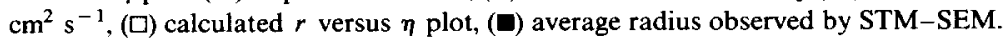

where $c$ is the surface concentration of mobile species per square centimeter. For an electrochemical system the maximum columnar growth rate is

$\partial h / \partial t=(M / \rho)\left(J_{\mathrm{M}} / z F\right)$,

so that

$A=M J_{\mathrm{M}} / z F c$.

By introducing eq. (25) into eq. (22), $R$ becomes

$R=(h / 3 \sqrt{2}) \sqrt{M J_{\mathrm{M}} / z F c D}$.

From eq. (26), by using the $J_{\mathrm{M}}$ values derived from the transients, (fig. 1), $h=2.3 \times 10^{-5} \mathrm{~cm}$ (eq. (13)) $D=7 \times 10^{-12} \mathrm{~cm}^{2} \mathrm{~s}^{-1}$ and $c=7.3 \times 10^{-7} \mathrm{~g}$ $\mathrm{cm}^{-2}$, the latter estimated from the oxide density, the shape of the $R$ versus $\eta$ plot can be reproduced (fig. 9). Eq. (26) implies that the slope of the $\log R$ versus $\eta$ is twice that of the $\log J_{\mathrm{M}}$ versus $\eta$, a fact which has been experimentally verified (figs. $1 \mathrm{~b}$ and 2 ).

From eqs. (18), (20), (21) and (25) and $D=7 \times 10^{-12} \mathrm{~cm}^{2} \mathrm{~s}^{-1}$ the $r$ values can be calculated (fig. 9). These values of $r$ are in good agreement with those observed by STM-SEM.

The physical picture underlying this model implies that at low $\eta, A$ is small, so that after their arrival the metal ions can diffuse a relatively large distance over the surface before their incorporation to growing nuclei. It means that an electrodepositing particle can visit a large number of sites before it is finally incorporated into the metal lattice. Thus, the voids between monomers are filled and they coalesce to form large units which lead to a low $R$ value. As $\eta$ and $A$ increase, the diffusion path is reduced, so that the voids between monomers are not totally filled. The grain size decreases and accordingly $R$ increases. Finally at the highest $\eta$ values the rate of the interfacial reaction becomes sufficiently large so that the sites at the oxide layer adjacent to the growing metal surface become depleted of metal ions. The kinetics of 
the growth process approaches a transport control due to metal ions moving from the oxide phase to the metal overlayer. During the transport controlled electrodeposition, the arriving particles can diffuse only a very small distance on the metal surface before sticking. Thus, unoccupied sites become shadowed so that voids are trapped into the structure leading to a low density material. In this condition the coalescence process is not determinant and the structure exhibits the largest $R$ values. Monte Carlo simulation of surfaces growing under random diffusion and a sticking probability depending on $\eta$, gives the same results [5].

The self-shadowing and voids formation occur when the rate of diffusion of metal atoms to the shadowed regions is smaller than the rate of void incorporation via growth [27]. After completion of the phase change, the low density structure appears unstable. The decrease of $R$ with time is clearly connected with the decrease of the electrodeposit surface in order to achieve the lowest surface free energy [28].

The particle coalescence mechanism involved in this process [28] is controlled by surface diffusion of adatoms from higher to lower chemical potential i.e. from the small to the large particles. Accordingly large particles are formed by coalescence of small ones. Thus, the measurement of the time dependence of $R$ should be a simple and accurate method for measuring surface diffusion coefficients in electrochemical systems of particular interest for metal electrocrystallization and corrosion. This matter will be discussed in a forthcoming publication [29].

\section{Acknowledgments}

A fellowship from the Consejo Nacional de Investigaciones Cientificas y Tecnicas (Argentina) to R.C.S is gratefully acknowledged. Financial support was obtained from DGICT through contract number PB86-0606. We are indebted to A. Buendia for skillful technical assistance and to J. Gomez and J.M. Gomez for help in data acquisition and image processing.

\section{References}

[1] A.E. Bolzan, A.M. Castro Luna, A. Visentin, R.C. Salvarezza and A.J. Arvia, Electrochim. Acta 33 (1988) 1743.

[2] M.L.A. Temperini, O. Sala, G.E. Lacconi, A.S. Gioda, V.A. Macagno and A.J. Arvia, Langmuir, in press.

[3] M.E. Vela, R.C. Salvarczza and A.J. Arvia, Elcctrochim. Acta, submittcd.

[4] D.D. Tuschel and J.E. Pemberton, Langmuir 4 (1988) 58.

[5] E.A. Albano, H.O. Martin, R.C. Salvarezza, M.E. Vela and A.J. Arvia, J. Electrochem. Soc., in press. 
[6] S. Lichter and J. Chen, Phys. Rev. Letters 56 (1986) 1396.

[7] J.K. Gimzewski, A. Humbert, J.G. Bednorz and B. Reihl, Phys. Rev. Letters 55 (1985) 951.

[8] J. Gomez, L. Vazquez, A.M. Baro, C.L. Perdriel, W.E. Triaca and A.J. Arvia, Nature 323 (1986) 612.

[9] J. Gomez, L. Vazquez, A.M. Baro, C. Alonso, E. Gonzalez, J. Gonzalez-Velasco and A.J. Arvia, J. Electroanal. Chem. 240 (1988) 77.

[10] L. Vazquez, J. Gomez, A.M. Baro, N. Garcia, M.L. Marcos, J. Gonzalez-Velasco, J.M. Vara, J. Presa, A. Garcia, M. Aguilar and A.J. Arvia, J. Am. Chem. Soc. 109 (1987) 1730.

[11] Ch. Gerber, G. Binnig, H. Fuchs, O. Marti and H. Rohrer, Rev. Sci. Instr. 57 (1986) 221.

[12] T. Ichinokawa, Y. Miyazaki and Y. Koga, Ultramicroscopy 23 (1987) 115.

[13] M. Anders, M. Thaer, M. Mück and C. Heiden, J. Vacuum Sci. Technol. A 6 (1988) 436.

[14] L. Vazquez, A. Bartolome, R. Garcia, A. Buendia and A.M. Baro, Rev. Sci. Instr. 59 (1988) 1286.

[15] A. Bartolome, R. Garcia, L. Vazquez and A.M. Baro, J. Microsc., in press.

[16] A. Chialvo, W.E. Triaca and A.J. Arvia, J. Electroanal. Chem. 171 (1984) 303.

[17] L. Vazquez, A. Bartolome, A.M. Baro, C. Alonso, R.C. Salvarezza and A.J. Arvia, Extended Abstract of the 174th Meeting of The Electrochemical Society, Chicago, Illinois, October 9-14 (1988).

[18] A.J. Bard, Encyclopedia of Electrochemistry of the Elements, Vol. 4 (Dekker, New York, 1975) p. 87.

[19] M. Fleischmann and H.R. Thirsk, in: Advances in Electrochemistry and Electrochemical Engineering, Vol. 3, Ed. P. Delahay (Interscience, New York, 1961) p. 123.

[20] H.R. Thirsk and J. Harrison, A Guide to Study of Electrode Kinetics (Academic Press, New York, 1972).

[21] B. Scharifker and G.J. Hills, Electrochim. Acta 28 (1983) 879.

[22] J. Mostany, J. Mozota and B. Scharifker, J. Electroanal. Chem. 177 (1984) 25.

[23] D.M. Kolb and J. Schneider, Electrochim. Acta 31 (1986) 929.

[24] A. Hamelin, Electrochim. Acta 31 (1986) 937.

[25] L. Vazquez, A. Bartolome and A.M. Baro, in preparation.

[26] D. Dickertmann, J.W. Schultze and K.J. Vetter, J. Electroanal. Chem. 55 (1974) 429.

[27] H.J. Leamy, G.H. Gilmer and A.G. Dirks, in: Current Topics in Materials Science, Vol. 6, Ed. E. Kaldis (North-Holland, Amsterdam, 1980) p. 311.

[28] H.P. Bonzel, in: Surface Physics of Materials, Ed. J.M. Blakely (Academic Press, New York, 1975) p. 280.

[29] L. Vazquez, A. Bartolome, A.M. Baro, C. Alonso, K.C. Salvarezza and A.J. Arvia, in preparation. 